\title{
Dynamical Stability and Habitability of Extra-Solar Planets
}

\author{
Elke Pilat-Lohinger
}

Institute of Astronomy, University of Vienna,

Türkenschanzstrasse 17, A-1180 Vienna, Austria

email: elke.pilat-lohinger@univie.ac.at.

\begin{abstract}
Observations of about 60 binary star systems hosting exoplanets indicate the necessity of stability studies of planetary motion in such multi-stellar systems. For wide binary systems with separations between hundreds and thousands of AU, the results from single-star systems may be applicable but, in tight double stars systems, we have to take the stellar interactions into account which influences the planetary motion significantly.

This review discusses the different types of planetary motion in double stars and the stability of the planets for different binary configurations. An application to the most famous tight binary system ( $\gamma$ Cephei) is also shown. Finally, we analyze the habitability from the dynamical point of view in such systems, where we discuss the motion of terrestrial-like planets in the so-called habitable zone.
\end{abstract}

Keywords. Binaries: general, planetary systems, n-body simulations, numerical

\section{Introduction}

Stability studies of planetary motion in binary star systems are very important, since we expect an increasing number of detected planets in multi-stellar systems in the future - due to the fact that most of the stars in the solar neighborhood form double or multiple star systems (see e.g. Duquenor \& Mayor 1991). From the dynamical point of view, we distinguish 3 types of motion in such systems (Dvorak 1984):

(i) S-type motion, where the planet moves around one stellar component;

(ii) P-type motion, where the planet surrounds both stars in a very distant orbit; and

(iii) L-type motion, where the planet moves in the same orbit as the secondary. They are locked in 1:1 mean motion resonance, which makes the system stable. But, the stability is limited to certain mass-ratios of the two stars: $\mu=m_{2} /\left(m_{1}+m_{2}\right)<1 / 26$. Therefore, this motion is not so interesting for double stars.

The literature shows that long before the first planet in a binary system was discovered, astronomers, working in Dynamical Astronomy, carried out theoretical and numerical stability studies for the different types of motion (see e.g. Dvorak 1984, 1986; Rabl \& Dvorak 1988; Dvorak et al. 1989). The discovery of planets in such systems convinced many research groups to examine the planetary formation and evolution in binary star systems, either in general or for selected ones (see e.g. Holman et al. 1997; Holman \& Wiegert 1999; Ford et al. 2000; Pilat-Lohinger \& Dvorak 2002; Pilat-Lohinger et al. 2003; Dvorak et al. 2003a,b; Thébault et al. 2010; Musielak et al. 2005; Haghighipour 2006; Raghavan et al. 2006; Cuntz et al. 2007; Kley \& Nelson 2008; Paardekooper et al. 2008; Takeda et al. 2008; Saleh \& Rasio 2009; Marzari et al. 2010; Haghighipour et al. 2010; and many others).

Most of the planets detected so far are in S-type (or circumprimary) motion, so that we will mainly discuss this type of motion in this paper. Although, planets moving in 
P-type (or circumbinary) orbits have been detected during the last few months, we learned during this symposium that the existence of these planets is still quite questionable. Dynamical studies of P-type motion by Dvorak et al. (1989), Holman \& Wiegert (1999), Pilat-Lohinger et al. (2003) have shown that a planetary orbit surrounding both stars is only stable for distances (from the mass-center) larger than twice the separation of the two stars. In the case of eccentric motion of the binary, the planet's distance to the mass-center has to be increased in order to be stable.

In the following sections, we will discuss the stability of circumprimary motion in general, taking into account the eccentricity of the binary and of the planet. Moreover, we show the influence of the planet's mass on the stable region and apply the study to the most famous tight binary star system that hosts a planet (i.e. $\gamma$ Cephei).

\section{Dynamical model and computations}

Most of the studies mentioned above used for the numerical simulations the elliptic restricted three body problem (ER3BP) that describes the motion of a mass-less body in the gravitational field of two massive bodies, which move in elliptic orbits (Keplerian motion) around their center of mass, without being influenced by the mass-less body. Numerical studies by Rabl \& Dvorak (1988; hereafter, RD) and Holman \& Wiegert (1999; hereafter, HW) determined the stable region as a function of the binary's mass-ratio and eccentricity, and the motion of the planet was circular The numerical investigation by Pilat-Lohinger \& Dvorak (2002; hereafter, PLD) also analyzed the influence of the planet's eccentricity. In these three investigations, the stable regions of planetary motion have been determined in a similar way. The planet (which is considered as mass-less body) moves around star $m_{1}$. The distance between $m_{1}$ and the second star $\left(m_{2}\right)$ is set to 1 , and the eccentricity of the binary varies between 0 and 0.9 with a step of 0.1 . There are two starting positions for $m_{2}$ : the peri-center and the apo-center.

The planet's semi-major axis is taken between 0.1 and 0.9 . For each orbit, at least four starting positions were used (i.e. mean anomaly $=0^{\circ}, 90^{\circ}, 180^{\circ}, 270^{\circ}$ ) and the planet's eccentricity was set to zero in RD and HW, and was varied between 0 and 0.5 (or 0.9 in certain cases) with a step of 0.1 for all mass-ratios in PLD.

Moreover, in PLD, the orbital behavior was determined by means of the Fast Lyapunov Indicator (FLI; see Froeschle et al. 1997), which is quite a fast tool to distinguish between regular and chaotic motion. Chaotic orbits can be found very quickly because of the exponential growth of the tangent vector in the chaotic region. For most chaotic orbits, only a small number of primary revolutions is needed to determine the orbital behavior. In order to distinguish between stable and chaotic motion, we defined a critical value for the FLIs depending on the computation time. In the general stability study of S-type motion, the FLIs were computed for 1000 periods of the binary. $\dagger$ A comparison of the results of RD, HW and PLD show them in good agreement. Minor variations are caused by the different methods used to determine the stable region $\ddagger$. Table 1 shows the border of stable motion for circular S-type orbits in binary star systems for different mass-ratios and different eccentricities of the two stars. This border is defined by the largest distance of the planet to its host-star $m_{1}$, where stable motion has been found for all initial positions of the planet.

$\dagger$ Even if the computation time seems to be quite short, one has to take into account that the results are valid for a much longer time due to the application of the FLI, and test-computations of three selected mass-ratios over a longer time (of $10^{4}, 10^{5}$ and $10^{6}$ primary periods) did not change the result significantly.

$\ddagger$ In some cases the FLI results indicates a slightly larger stable region due to the fact that only 4 starting positions were used whereas HW used 8 . 
Table 1. Stable zone (in dimensionless units) of S-type motion for all computed mass-ratios and eccentricities of the binary. The given size for each $\mu, e_{b i n a r y}$ pair is the lower value of the studies by HW and PLD.

\begin{tabular}{|c|c|c|c|c|c|c|c|c|c|}
\hline & \multicolumn{9}{|c|}{ mass-ratio $(\mu)$} \\
\hline | $e_{\text {binary }} \mid$ & 0.1 & 0.2 & 0.3 & 0.4 & 0.5 & 0.6 & 0.7 & 0.8 & 0.9 \\
\hline 0.0 & 0.45 & 0.38 & 0.37 & 0.30 & 0.26 & 0.23 & 0.20 & 0.16 & 0.13 \\
\hline 0.1 & 0.37 & 0.32 & 0.29 & 0.27 & 0.24 & 0.20 & 0.18 & 0.15 & 0.11 \\
\hline 0.2 & 0.32 & 0.27 & 0.25 & 0.22 & 0.19 & 0.18 & 0.16 & 0.13 & 0.10 \\
\hline 0.3 & 0.28 & 0.24 & 0.21 & 0.18 & 0.16 & 0.15 & 0.13 & 0.11 & 0.09 \\
\hline 0.4 & 0.21 & 0.20 & 0.18 & 0.16 & 0.15 & 0.12 & 0.11 & 0.10 & 0.07 \\
\hline 0.5 & 0.17 & 0.16 & 0.13 & 0.12 & 0.12 & 0.09 & 0.09 & 0.07 & 0.06 \\
\hline 0.6 & 0.13 & 0.12 & 0.11 & 0.10 & 0.08 & 0.08 & 0.07 & 0.06 & 0.045 \\
\hline 0.7 & 0.09 & 0.08 & 0.07 & 0.07 & 0.05 & 0.05 & 0.05 & 0.045 & 0.035 \\
\hline 0.8 & 0.05 & 0.05 & 0.04 & 0.04 & 0.03 & 0.035 & 0.03 & 0.025 & 0.02 \\
\hline
\end{tabular}

As already mentioned, the study by PLD examined also eccentric planetary motion in the different binary configurations and showed that the reduction of the stable zone due to an increase of the binary's eccentricity is certainly stronger than the influence of the planet's eccentricity. Fig. 1 shows a summary of this study for $\mu=0.2$, where we see for each $\left(e_{\text {Binary }}, e_{\text {Planet }}\right)$ pair on the $(\mathrm{x}, \mathrm{y})$ plane the respective extension of the stable zone (z-axis), which is defined by the semi-major axis of the last stable orbit (corresponding to the largest distance of the planet to its host-star). The Gray plane represents the limiting plane for stable motion. Similar 3-D plots for all mass-ratios (from 0.1 to 0.9 ) as well as a detailed discussion of the results are given in PLD.

Even if the size of the stable region does not show a strong dependence on the eccentricity of the planet, it is not negligible, especially if a planet is close to the border of chaotic motion and moves in a highly eccentric orbit. In a recent study, where we examined the influence of the planetary mass on the size of the stable region, we found out that eccentric motion of massive planets would shrink the stable zone significantly (a paper thereto is in preparation).

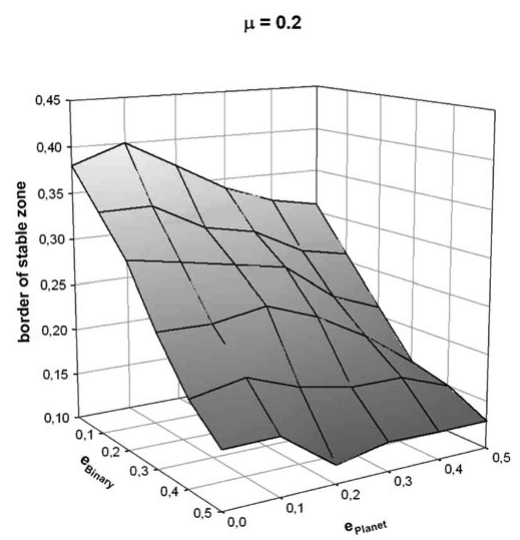

Figure 1. The size of the stable zone of S-type motion in a binary with mass-ratio $\mu=0.2$ depending on the eccentricity of the binary (x-axis) and of the planet (y-axis) It is clearly seen that the variation of $e_{\text {Binary }}$ influences the extension of the stable zone (z-axis) stronger than the variation of $e_{\text {Planet }}$. 
In the next section, we show the application of the results for $\mu=0.2$ given in Fig. 1 to the binary $\gamma$ Cephei, where a giant planet has been discovered by Hatzes et al. (2003).

\section{Planetary motion in the binary $\gamma$ Cephei}

$\gamma$ Cephei is one of the most interesting double star systems that hosts a planet. It is nearly 14 pc away from our Solar System and consists of a K1 IV star (of $1.4-1.6$ solar masses) and a M4 V star (of 0.4 solar masses). Thus, the mass-ratio of this system is about 0.2 . The detected planet of $1.76-1.85$ Jupiter-masses orbits the K1 IV star at a distance of about $2 \mathrm{AU}$.

Applying the general stability study, the border of stable planetary motion is between 3.6 and $3.8 \mathrm{AU}$, which shows that the detected planet is obviously in the stable region in case of circular planetary motion. If we increase the planet's eccentricity up to 0.5 , the stable region will shrink to less than 3 AU. Taking into account different masses for the planet, the reduction of the stable region strongly depends on the mass and the eccentricity of the planet: for a 1 Jupiter-mass $(=\mathrm{JM})$ planet, the stable region is reduced by $1.6 \mathrm{AU}$; for a $3 \mathrm{JM}$ planet, the reduction is $1.8 \mathrm{AU}$ and for a $5 \mathrm{JM}$ planet, $2.2 \mathrm{AU}$ if we increase the planet's eccentricity from 0 to 0.5 . This shows clearly, that a massive planet moving in a high eccentric orbit can easily be dropped out of the stable region determined for circular planetary motion by HW or RD.

\section{Binary star systems and habitable planets?}

The habitable zone (HZ) is the region around a star, where liquid water is stable on the surface of an Earth-like planet (see Kasting et al. 1993). Another assumption for such a planet is the existence of an appropriate planetary atmosphere. The study of habitability is certainly an interdisciplinary venture including astrophysical, biological, geophysical, and chemical studies. From the astrophysical point of view, studies of the stellar luminosity and its influence on the distance of the HZ as well as the planet's mass (to maintain an atmosphere) and planetary composition (assuming a terrestrial planet) are important contributions to the science of habitability. It is well known, that the evolution of a biosphere is a process over a long time, therefore, it is obvious that longterm orbital stability of a planet in the HZ represents one of the basic requirements for habitability. This emphasizes the importance of such numerical investigations for known and future extra-solar planetary systems. Using the boundaries of the HZ as defined by Kasting et al. (1993), the size of this zone is limited to a small region, depending on the spectral type and the age of the host-star. Taking into account the studies by Forget et al. (1997) or Mischna et al. (2000), we get a potentially larger HZ for a sun-like star. However, the planet's eccentricity has to be small enough if we require that the whole orbit of a planet is in the HZ. In dynamical studies, we distinguish different types of HZ, depending on the location of the giant planet in the system:

(1) The inner HZ, where the HZ is between the host-star and the detected giant planet.

(2) The outer HZ, where the HZ is outside the giant planet (in case of hot-Jupiters).

(3) The giant-planet habitable zone (GHZ) where the detected giant planet moves in the HZ. In this case, we can only expect so-called habitable moons or habitable Trojan planets (the latter corresponds to L-type motion; see Laughlin \& Chambers 2002; Dvorak et al. 2004; or Erdi \& Sandor 2005).

In the case of a binary star system, the situation is more difficult since we have to study as well the influence of the second star on the planetary motion. Considering a second planet in the binary $\gamma$ Cephei, which moves between the host-star and the detected 

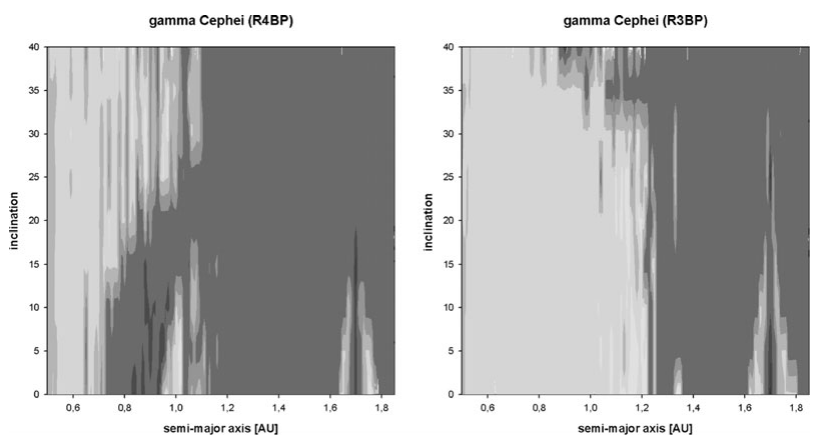

Figure 2. FLI stability maps for a fictitious planet in the vicinity of $\gamma$ Cephei: left panel shows the result in the restricted 4 body problem (R4BP) (i.e. $m_{1}+m_{2}+$ detected planet + fictitious planet) and right panel shows the result in the restricted 3 body problem (R3BP) (i.e. $m_{1}+$ detected planet + fictitious planet). The dark region shows the chaotic zone and the white area the stable one.

giant planet, the influence of the M4 V star at about 20 AU can be visualized by doing calculations with (left panel of Fig. 2) and without (right panel of Fig. 2) this star. A comparison of the two results shows significant differences. The presence of the perturbing star (see Fig. 2.a) decreases the stable region (i.e. the faint region in the panels) and shows an arc-like chaotic structure with a stable island around $1 \mathrm{AU}$ (which corresponds to the 3:1 mean motion resonance). A first study about this significant difference is given in Pilat-Lohinger (2005), where a variation of the semi-major axis of the detected giant planet shows the following:

When the giant planet is quite close to the host-star (e.g. around 1.3 AU) the faint region in Fig. 2 is mainly perturbed by mean motion resonances with respect to the giant planet. If the giant planet is shifted towards the M4 V star, the curved chaotic structure appears due to secular perturbations, because this star causes a precession of the perihelion of the giant planet. (As all massive bodies were placed in the same plane a precession of the ascending node cannot be modeled.)

Since the host-star $\gamma$ Cephei is already a sub-giant and the detected giant planet moves in the HZ, we used for the our numerical study of habitability from the dynamical point of view another real binary star system HD41004 AB, where a giant planet has been discovered by Zucker et al. (2003 and 2004). Using various configurations (real and fictitious) of this binary and the detected giant planet, and adding a fictitious terrestrial planet between HD41004 A and the giant planet, we have seen that certain configuration would allow the existence of a habitable planet, at least from the dynamical point of view - i.e. long-term stability of the whole system and low-eccentricity motion of the planet in the HZ. A first result is given in Pilat-Lohinger \& Funk (2010) and a more detailed study is in preparation.

Of course this result is just "a small part of a big puzzle," but it is well known that the long-term stability of a planetary system is one of the basic requirements for habitability. Since our dynamical studies have shown that potential habitable planets could also exist in binary star systems, the study of habitability in multi-star systems is certainly an interesting topic for future research. Especially, as we know that most of the stars in the solar neighborhood are in double or multiple star systems.

\section{Acknowledgements}

EP-L acknowledges the support by the Austrian Science Fund (FWF) - project no. P22603-N16. 


\section{References}

Cuntz, M., Eberle J., \& Musielak, Z. E. 2007, ApJ, 669, 105

Duquennoy, A. \& Mayor, M. 1991, A\&A, 248, 485

Dvorak, R. 1984, CMDA, 34, 369

Dvorak, R. 1986, A\&A A, 167, 379

Dvorak, R., Froeschlé, Ch., \& Froeschlé, C. 1989, A\&A, 226, 335

Dvorak, R., Pilat-Lohinger, E., Funk, B., \& Freistetter, F. 2003, A\& 4 , 398, L1

Dvorak, R., Pilat-Lohinger, E., Funk, B., \& Freistetter, F. 2003, A\&A A, 410, L13

Dvorak, R., Pilat-Lohinger, E., Schwarz, R., \& Freistetter, F. 2004, A\&\&A, 426, L37

Érdi, B. and Sándor, Zs. 2005, CMDA, 92, 113

Forget, F. \& Pierrehumbert, R. T. 1997, Science, 278, 1273

Froeschlé, C., Lega, E., \& Gonczi, R. 1997, CMDA, 67, 41

Haghighipour, N. 2006, ApJ, 644, 543

Haghighipour, N., Dvorak, R., \& Pilat-Lohinger, E. 2010, Planets in Binary Star Systems, ed. Haghighipour, N., ASS Library, 366, 285

Hatzes, A. P., Cochran, W. D., Endl, M., \& McArthur, B., et al. 2003, ApJ, 599, 1383

Holman, M. J., Touma, J., \& Tremaine, S. 1997, Nature, 386, 254

Holman, M. J. \& Wiegert P. A. 1999, AJ, 117, 621

Kasting, J. F., Whitmire D. P., \& Reynolds, R. T. 1993, Icarus, 101, 108

Kley, W. \& Nelson, R. P. 2008, A\&A, 487, 671

Laughlin, G. \& Chambers, J. E. 2002, AJ, 124, 592

Marzari, F., Thbauld, P., Kortenkanmp, S., \& Scholl, H. 2010, Planets in Binary Star Systems ed. Haghighipour, ASS Library 366, 165

Mischna, M. A., Kasting, J. F., Pavlov, A., \& Freedman, R. 2000, Icarus, 145, 546

Musielak, Z. E., Cuntz, M., Marshall, E. A., \& Stuit, T. D. 2005, A\&AA, 434, 355

Paardekooper, S. J., Thbault, P., \& Mellema, G. 2008, MNRAS, 386, 973

Pilat-Lohinger, E. \& Dvorak, R. 2002, CMDA, 82, 143

Pilat-Lohinger, E., Funk, B., \& Dvorak, R. 2003, A\&A, 400, 1085

Pilat-Lohinger, E. 2005, IAU Coll. 197, eds. Knezevic \& Milani, Cambridge Univ. Press, 71

Pilat-Lohinger, E. \& Dvorak, R. 2007, Extrasolar Planets, ed. Dvorak, Wiley-VCH, 179

Pilat-Lohinger, E. \& Funk, B. 2010, LNP, 790, p 481

Rabl, G. \& Dvorak, R. 1988, A\&̈A, 191, 385

Raghavan, D., Henry, T. J., Mason, B. D., Subasavage, J. P., Jao, W.-C., Beaulieu, T. D., \& Hambly, N. C. 2006, ApJ, 646, 523

Saleh, L. A. \& Rasio, F. A. 2009, ApJ, 694, 1566

Takeda, G., Kita, R., \& Rasio, F. A. 2008, ApJ, 683, 549

Thbault, P., Marzari, F., \& Augereau, J. C. 2010, A\& A, 524, 13

Zucker, S., Mazeh, T., Santos, N. C., Udry, S., \& Mayor, M. 2003, A\&\&A, 404, 775

Zucker, S., Mazeh, T., Santos, N. C., Udry, S., \& Mayor, M. 2004, A\&\&A, 426, 695

\section{Discussion}

S. ZuCKER: In the case of HD41004 AB, we don't know the details of the wide orbit, which may be eccentric.

E. Pilat-Lohinger: Yes, the eccentricity of HD41004 AB is not known, but with our stability analysis, it was possible to make some restrictions for the eccentricity of the binary. Moreover, we studied for the binary and the detected giant planet different fictitious configurations to examine if there are configurations that could host a habitable planet. 